\title{
BMJ Open Sleep Apnea and Fetal Growth Restriction (SAFER) study: protocol for a pragmatic randomised clinical trial of positive airway pressure as an antenatal therapy for fetal growth restriction in maternal obstructive sleep apnoea
}

\author{
Alex Hincker, ${ }^{1}$ Jacob Nadler, ${ }^{2}$ Suzanne Karan, ${ }^{2}$ Ebony Carter, ${ }^{3}$ Shay Porat, ${ }^{4}$ \\ Barbara Warner, ${ }^{5}$ Yo-El S Ju, ${ }^{6}$ Arbi Ben Abdallah, ${ }^{1}$ Elizabeth Wilson, ${ }^{1}$ \\ Ellen M Lockhart, ${ }^{1}$ Yehuda Ginosar (D) , ${ }^{1,7}$ the SAFER study group
}

To cite: Hincker A, Nadler J, Karan S, et al. Sleep Apnea and Fetal Growth Restriction (SAFER) study: protocol for a pragmatic randomised clinical trial of positive airway pressure as an antenatal therapy for fetal growth restriction in maternal obstructive sleep apnoea. BMJ Open 2021;11:e049120. doi:10.1136/ bmjopen-2021-049120

- Prepublication history and additional supplemental material for this paper are available online. To view these files, please visit the journal online (http://dx.doi.org/10.1136/ bmjopen-2021-049120).

Received 19 January 2021 Accepted 25 May 2021

Check for updates

(C) Author(s) (or their employer(s)) 2021. Re-use permitted under CC BY-NC. No commercial re-use. See rights and permissions. Published by BMJ.

For numbered affiliations see end of article.

Correspondence to

Dr Yehuda Ginosar;

ginosar@wustl.edu

\section{ABSTRACT}

Introduction Fetal growth restriction (FGR) is a major contributor to fetal and neonatal morbidity and mortality with intrauterine, neonatal and lifelong complications. This study explores maternal obstructive sleep apnoea (OSA) as a potentially modifiable risk factor for FGR. We hypothesise that, in pregnancies complicated by FGR, treating mothers who have OSA using positive airway pressure (PAP) will improve birth weight and neonatal outcomes.

Methods and analysis The Sleep Apnea and Fetal Growth Restriction study is a prospective, block-randomised, single-blinded, multicentre, pragmatic controlled trial. We enrol pregnant women aged 18-50, between 22 and 31 weeks of gestation, with established FGR based on second trimester ultrasound, who do not have other prespecified known causes of FGR (such as congenital anomalies or intrauterine infection). In stage 1, participants are screened by questionnaire for OSA risk. If OSA risk is identified, participants proceed to stage 2, where they undergo home sleep apnoea testing. Participants are determined to have OSA if they have an apnoea-hypopnoea index (AHI) $\geq 5$ (if the oxygen desaturation index (ODI) is also $\geq 5$ ) or if they have an $\mathrm{AHI} \geq 10$ (even if the $\mathrm{ODI}$ is $<5$ ). These participants proceed to stage 3 , where they are randomised to nightly treatment with PAP or no PAP (standard care control), which is maintained until delivery. The primary outcome is unadjusted birth weight; secondary outcomes include fetal growth velocity on ultrasound, enrolment-todelivery interval, gestational age at delivery, birth weight corrected for gestational age, stillbirth, Apgar score, rate of admission to higher levels of care (neonatal intensive care unit or special care nursery) and length of neonatal stay. These outcomes are compared between PAP and control using intention-to-treat analysis.

Ethics and dissemination This study has been approved by the Institutional Review Boards at Washington University in St Louis, Missouri; Hadassah Hebrew University Medical Center, Jerusalem; and the University of Rochester, New York. Recruitment began in Washington University in November 2019 but stopped from March to November
Strengths and limitations of this study

- Sleep Apnea and Fetal Growth Restriction study is a multicentre, pragmatic trial conducted in a tertiary care setting.

- Studying patients with both fetal growth restriction and home sleep apnoea testing-diagnosed obstructive sleep apnoea (OSA) increases the potential for benefit from the intervention but limits generalisability.

- The intervention is positive airway pressure, a routine, safe and effective therapy for OSA in nonpregnant populations.

- While investigators and care providers are blinded to group allocation in stage 3, participants are unblinded.

- Positive findings will increase OSA awareness and treatment in pregnancy.

2020 due to COVID-19. Recruitment began in Hadassah Hebrew University in March 2021, and in the University of Rochester in May 2021. Dissemination plans include presentations at scientific conferences and scientific publications.

Trial registration number NCT04084990.

\section{BACKGROUND}

Fetal growth restriction (FGR) affects up to $10 \%$ of all pregnancies and is a major contributor to fetal and neonatal morbidity and mortality with intrauterine, neonatal and lifelong complications. ${ }^{12}$ FGR is second only to prematurity as a leading cause of perinatal morbidity and mortality. Both FGR and prematurity are independent risk factors for the development of cognitive delay, poor academic achievement and adult diseases 
such as obesity, type 2 diabetes mellitus, coronary artery disease and stroke. ${ }^{3}$ There are many potential causes of FGR, but in the absence of underlying genetic conditions, congenital anomalies or intrauterine infection, FGR is typically due to impaired uteroplacental perfusion. ${ }^{24}$ Current assessment is based on repeated ultrasound assessments of fetal growth, antenatal testing including non-stress test and/or biophysical profile (including electronic fetal heart rate monitoring) and umbilical artery Doppler velocimetry. ${ }^{5}$ Intervention is limited to antenatal steroid administration and interventional delivery when the risk of stillbirth is deemed too high to continue the pregnancy. There is no intervention currently available to improve uteroplacental blood flow and fetal growth in utero, so there is often no alternative to interventional delivery. ${ }^{6}$ When the pregnancy is remote from term, interventional delivery exposes an already compromised fetus to additional complications of prematurity, in particular to neonatal brain injury from intraventricular haemorrhage. ${ }^{78}$

Pregnancy is associated with a higher incidence of sleep disordered breathing (SDB), a group of chronic conditions involving recurrent episodic partial or complete cessation of breathing throughout the night. ${ }^{9}$ Obstructive sleep apnoea (OSA) is an increasingly common form of $\mathrm{SDB}$ in both the general and pregnant population. ${ }^{10} \mathrm{OSA}$ is characterised by complete (apnoea) or incomplete (hypopnoea) collapse of the upper airway during sleep leading to recurrent episodic cessation or limitation of normal breathing. This in turn leads to recurrent oxygen desaturation and hypercapnia, frequent night-time arousals and excessive daytime sleepiness. ${ }^{11} 12$

OSA in pregnancy has been associated with poor maternal-fetal outcomes, including gestational hypertension, pre-eclampsia, gestational diabetes, FGR, low birth weight, preterm delivery and higher rates of neonatal intensive care unit (NICU) admission. ${ }^{13-20}$ Patients with OSA are more likely to have negative neonatal outcomes, ${ }^{21}$ and severe maternal morbidity and mortality, ${ }^{17}$ regardless of their obesity status. The relationship between severity of OSA in pregnancy and adverse outcomes is an ongoing area of research. ${ }^{22}$ Unfortunately, because few pregnant women are referred for polysomnography (PSG), it is likely that OSA and other sleep disorders are underdiagnosed, ${ }^{23}$ with the OSA-related symptoms of snoring, disrupted sleep and fatigue being frequently attributed to transient features of normal pregnancy. ${ }^{24}$

Recurrent apnoeic and hypopnoeic episodes are associated with intermittent oxygen desaturation and hypercapnia. Recurrent hypoxia leads to oxidative stress, sympathetic activation and inflammation that may be harmful to both the mother and her fetus. ${ }^{25}$ Acute hypercapnia in pregnant mice and rats has been shown to cause acute placental hypoperfusion and acute fetal asphyxia. ${ }^{26}$ There is very little information about the effect of these episodes on the human fetus. One small observational study demonstrated fetal heart rate decelerations accompanying maternal oxyhaemoglobin desaturation, ${ }^{27}$ while another found no association. ${ }^{28}$

Positive airway pressure (PAP) is a common and effective treatment for OSA which acts by mechanically splinting the upper airway with pressurised air to prevent collapse. PAP delivered at a single pressure (continuous PAP or CPAP) usually requires a sleep study to identify optimal settings; it also cannot respond to changes in upper airway function related to changes in habitus, body position, sleep stage or other factors. In contrast, autotitrating PAP (aPAP) can detect upper airway resistance and respond by adjusting the delivered pressure (within a selected range). The delivered pressures from aPAP can therefore be lower than from CPAP, which makes aPAP more tolerable for most patients. ${ }^{29}$ We use aPAP as the intervention for all patients in the Sleep Apnea and Fetal Growth Restriction (SAFER) study. However, as much of the medical literature is still based on CPAP rather than aPAP, we use the generic term PAP throughout the remainder of this manuscript.

PAP is a proven low-risk therapeutic intervention for patients with OSA in the general, non-obstetric population. Multiple studies have shown that PAP use in patients with OSA reduces the incidence of death from cardiacrelated complications including congestive heart failure, coronary artery disease, arrhythmia and stroke, ${ }^{30-32}$ and may improve outcomes related to diabetes. ${ }^{33} 34$ Small studies of PAP in pregnant women with severe preeclampsia and OSA diagnosed by PSG demonstrated improved maternal haemodynamic profiles and cardiac output $^{35}{ }^{36}$ although no obstetric or neonatal outcomes were measured.

The hypothesis of the SAFER study is that, in pregnancies complicated by FGR where the mothers have been diagnosed with OSA, maternal PAP therapy will improve intrauterine fetal growth and birth weight, increase randomisation-to-delivery interval and the gestational age at delivery and improve neonatal well-being. Ultimately, if PAP therapy is shown to improve intrauterine fetal growth in pregnancies with OSA and FGR, obstetric practice would be expected to change. Such a finding would lead to a clinical imperative to screen, diagnose and treat OSA in pregnancy, particularly in the presence of FGR.

\section{METHODS \\ Research design overview}

The Human Research Protection Office at Washington University School of Medicine in St Louis, Missouri, the Research Subject Review Board at the University of Rochester, New York, and the Helsinki Committee for Ethics in Research in Human Subjects in Hadassah Hebrew University Medical Center, Jerusalem, Israel, all approved the study. The choice of these sites was determined by the location of the principal investigators involved in initiation of this study; however, all sites are academic, highrisk obstetric, tertiary referral centres with large numbers of pregnancies complicated by FGR. There is wide 


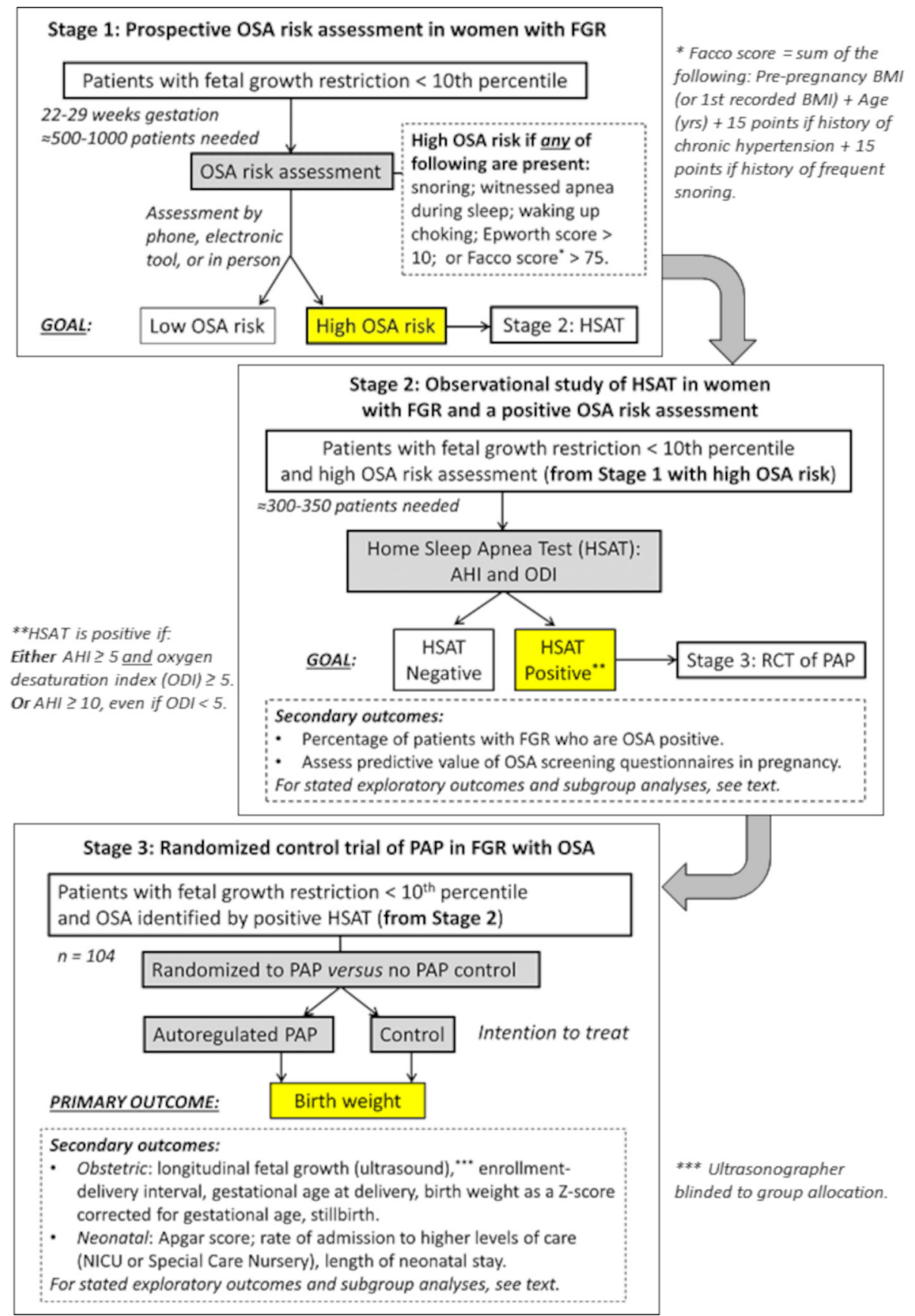

Figure 1 Sleep Apnea and Fetal Growth Restriction (SAFER) study flow chart. AHI, apnoea-hypopnoea index; BMI, body mass index; FGR, fetal growth restriction; NICU, neonatal intensive care unit; OSA, obstructive sleep apnoea; PAP, positive airway pressure; $\mathrm{RCT}$, randomised controlled trial.

geographic and demographic diversity between these centres, which adds to the generalisability of the study. The SAFER study detailed in this protocol adheres to the Standard Protocol Items: Recommendations for Interventional Trials checklist, ${ }^{37}$ and lists the 24 items in the WHO Trial Registration Data Set. The protocol version is dated 5 February 2020. The SAFER study is a three-stage trial that assesses pregnant patients with diagnosed FGR. The overall flow of participants through the SAFER study is shown in figure 1.

We enrol women with pregnancies complicated by FGR between 22 and 31 weeks of gestation. Stage 1 is a brief telephone or in-person questionnaire to identify which of these pregnant women are at elevated risk for OSA; these women are then recruited into stage 2. Stage 2 is a prospective observational study using home sleep apnoea testing (HSAT) to confirm the diagnosis of OSA. Participants meeting OSA diagnostic criteria progress to stage 3 . Stage 3 is a pragmatic randomised clinical trial of PAP as the intervention, versus a control group of standard care which does not include PAP. The primary outcome of stage 3 is birth weight; secondary outcomes include fetal growth velocity on ultrasound, birth weight corrected for gestational age, enrolment-to-delivery 
Table 1 Demographic characteristics of the medical centres in the SAFER study (data for 2019)

\begin{tabular}{|c|c|c|c|c|c|}
\hline Institution & Deliveries & CS rate (\%) & $\begin{array}{l}\text { US protocol used for } \\
\text { estimated fetal weight }\end{array}$ & $\begin{array}{l}\text { US fetal growth nomogram } \\
\text { used for FGR }\end{array}$ & $\begin{array}{l}\text { Birthweight nomogram } \\
\text { used for SGA }\end{array}$ \\
\hline $\begin{array}{l}\text { University of Washington, } \\
\text { Barnes Jewish Hospital, St } \\
\text { Louis, Missouri }\end{array}$ & 3576 & 31.4 & Hadlock et $a^{55}$ & $\begin{array}{l}\text { A US national reference for fetal } \\
\text { growth. }{ }^{56}\end{array}$ & $\begin{array}{l}\text { Revised Fenton growth chart } \\
\text { for preterm infants. } .^{57}\end{array}$ \\
\hline $\begin{array}{l}\text { University of Rochester, } \\
\text { Strong Hospital, Rochester, } \\
\text { New York }\end{array}$ & 2859 & 34.9 & Hadlock et al ${ }^{58}$ & $\begin{array}{l}\text { Local nomogram from } 115000 \\
\text { births in the nine-county Finger } \\
\text { Lakes Region, 2004-2013. } \\
60 \text { (For Rochester birthweight } \\
\text { nomogram see online } \\
\text { supplemental file 2). }\end{array}$ & $\begin{array}{l}\text { Local nomogram from } \\
115000 \text { births in the } \\
\text { nine-county Finger Lakes } \\
\text { Region, 2004-2013. }{ }^{59} 60 \\
\text { (For Rochester birthweight } \\
\text { nomogram see online } \\
\text { supplemental file 2). }\end{array}$ \\
\hline \multirow[t]{2}{*}{$\begin{array}{l}\text { Hadassah Hebrew University } \\
\text { Medical Center, Jerusalem, } \\
\text { Israel }\end{array}$} & $\begin{array}{l}8050 \\
\text { Ein Karem } \\
\text { campus }\end{array}$ & 17.1 & Hadlock et a $\left.\right|^{58}$ & $\begin{array}{l}\text { International estimated fetal } \\
\text { weight standards of the } \\
\text { INTERGROWTH-21st Project. }\end{array}$ & $\begin{array}{l}\text { Birthweight standards in the } \\
\text { liveborn population in Israel. }{ }^{62}\end{array}$ \\
\hline & $\begin{array}{l}5812 \\
\text { Mt Scopus } \\
\text { campus }\end{array}$ & 16.8 & & & \\
\hline
\end{tabular}

CS, caesarean section; FGR, fetal growth restriction; SAFER, Sleep Apnea and Fetal Growth Restriction; SGA, small for gestational age; US, ultrasound.

interval, gestational age at delivery and neonatal outcomes.

All three stages of this international, multicentre study will be conducted at three academic medical centres, which all serve a large and diverse population of highrisk obstetric patients. These centres are: Washington University School of Medicine in St Louis, Missouri (Barnes-Jewish Hospital); University of Rochester, New York (Strong Memorial Hospital); and Hadassah Hebrew University Medical Center, Jerusalem, Israel (Hadassah Hospital, Ein Karem and Hadassah Hospital, Mt Scopus). The demographic characteristics of these hospitals are summarised in table 1.

There is lack of clarity in current guidelines over the use of the term FGR. We use the American College of Obstetrics and Gynecology definition of FGR as estimated fetal weight below the 10 th percentile for a population, ${ }^{5}$ and small for gestational age (SGA) as birth weight below the 10th percentile.

\section{Patient and public involvement}

In an unpublished pilot study completed prior to completion of this protocol, pregnant volunteers used HSAT as described in stage 2, and OSA-positive subjects used PAP as described in stage 3 . The pilot study allowed investigators to assess the night-time tolerability of both HSAT and PAP for participants. Out of 57 patients who received HSAT, 52 had data adequate for analysis. Of these 52 patients, 10 met OSA criteria for PAP use (see below); however, one had an urgent delivery before PAP could be distributed. Of the remaining nine subjects, one declined to use PAP, one could not tolerate it and all the remaining seven met PAP adherence criteria (see below). Generally, adherence and comfort were good for both HSAT and PAP.

As part of the effort to design the study with a tolerable intervention and a meaningful outcome, two of our principal investigators met with community obstetricians, primary care physicians and with a non-profit organisation advocating for high-risk pregnant maternal health, in order to discuss the study and obtain feedback well in advance of its final iteration.

\section{Study participants}

\section{Inclusion criteria}

This study enrols participants in a three-stage protocol. We enrol pregnant women, aged $18-50$ years, who have been diagnosed with established FGR. FGR is defined as estimated fetal weight $<10$ th percentile based on at least one routine second trimester ultrasound without a subsequent increase to $>15$ th percentile on any ultrasounds prior to enrolment. If the ultrasound that identified FGR was performed prior to 21 completed weeks, a repeat scan after 22 weeks is required to confirm the diagnosis of FGR prior to enrolment. The lower limit of gestational age at enrolment to stage 1 is $22+0$ weeks; the upper limit of gestational age at enrolment to stage 1 is an adequate gestational age to be able to complete stages 1 and 2 and, if appropriate, to receive stage 3 intervention by no later than $32+0$ weeks.

\section{Exclusion criteria}

The following exclusion criteria apply at the time of enrolment: prespecified independent cause of FGR (congenital or genetic anomalies, suspected aneuploidy with two minor or one major markers, intrauterine infection or multiple gestation); active labour; a concrete decision already made to induce labour or perform caesarean delivery within 2 days; reverse end-diastolic flow in the umbilical artery (note that other abnormal Doppler flow velocities such as absent end-diastolic flow in the umbilical artery or uterine artery notching or increased pulsatility index are not exclusion criteria unless other exclusion criteria are present); pre-existing formal diagnosis of OSA; chronic pulmonary disease; haemoglobinopathies (including thalassemia major and sickle cell disease, but 
not sickle trait or thalassemia minor without clinical manifestations); maternal craniofacial anomalies which might impair the ability of the participant to use PAP appliances; lack of proficiency in English (Rochester or St Louis) or either Hebrew, Arabic or English (Jerusalem); inability to understand the consent; and unwillingness or inability to participate adequately in OSA screening (stage 1) or in HSAT (stage 2). Note that poor adherence to PAP (stage 3 ) is not an exclusion criterion. PAP usage is recorded but outcomes are assessed by intention to treat.

\section{Stage 1: initial screening for OSA risk}

Stage 1 of the trial is designed in accordance with the Strengthening the Reporting of Observational Studies in Epidemiology (STROBE) statement (https://www. strobe-statement.org). Potential participants with FGR as defined above are identified through referral from the obstetric ultrasound service, directly from the obstetrician or by review of the electronic medical record. Those who meet inclusion and exclusion criteria are approached for consent either by telephone, in the outpatient clinics or as inpatients by a member of the research team. Participants undergo a brief screening questionnaire to assess for risk of OSA. Participants are deemed to be at risk for OSA if they report loud snoring, report witnessed gasping for air during sleep, have an Epworth score $>10$ or a Facco score $>75$ (see figure 1).$^{20}$ Participants with evidence of OSA risk are invited to proceed to stage 2.

\section{Stage 2: HSAT and OSA screening tools}

Participants identified as being at risk for OSA in stage 1 progress to stage 2. The primary assessment for confirming OSA diagnosis in this study is HSAT. Stage 2 of the trial is an observational study designed in accordance with the STROBE statement (https://www.strobestatement.org/).

Prior to HSAT, participants are assessed for demographic data, medical comorbidities, body mass index, neck circumference and the presence of any craniofacial abnormalities. They also undergo a more detailed OSA assessment consisting of component parts of five standard OSA screening tools (STOP-BANG, Berlin Questionnaire, American Society of Anesthesiologists checklist, Flemons Index and Epworth Sleepiness Scale). These screening tools for OSA have each been validated in the non-pregnant population; some but not all have been validated in pregnancy. ${ }^{20} 3839$

HSAT is performed with a Food and Drug Administration-approved type III home sleep monitor (ResMed Apnea Link Air, ResMed, San Diego, California), which records the following: (1) air flow using nasal cannula and pressure transducer; (2) respiratory effort using elastic respiratory inductance plethysmography belts around the chest and abdomen; (3) ECG; and (4) pulse oximetry. ${ }^{40}$ A trained member of the study team shows each study participant how to apply the sensors and use the HSAT monitor and instructs her to wear the HSAT monitor for two consecutive nights. The
HSAT monitor data are downloaded, and studies are reviewed and scored by a registered polysomnographic technologist per standard scoring protocols for apnoeahypopnoea index (AHI) and oxygen desaturation index (ODI). ${ }^{41} \mathrm{AHI}$ is defined as the number of apnoeas plus hypopnoeas on average each hour.

Hypopnoea is defined by the American Academy of Sleep Medicine recommended rule 1A; where a hypopnoea requires all of the following to be met: (1) the peak signal for respiratory excursions drops by $\geq 30 \%$ from pre-event baseline; (2) the duration of the $\geq 30 \%$ drop is $\geq 10 \mathrm{~s}$; and (3) there is a $\geq 3 \%$ oxygen desaturation from pre-event baseline and/or the event is associated with an arousal. ${ }^{41}$ ODI is defined as the number of $\geq 3 \%$ oxygen desaturations per hour of sleep without the required drop in peak signal excursions.

HSAT generally underestimates AHI compared with PSG, as the entire study duration is used as the denominator when calculating AHI. These errors are most evident in mild OSA. ${ }^{42}$ To maximise sensitivity to detect OSA yet improve specificity in this pragmatic study, we add an ODI $\geq 5$ criterion in cases where AHI is between 5 and 10. Accordingly, an HSAT that is positive for OSA is defined as either an AHI $\geq 5$ with an ODI $\geq 5$ or as an AHI $\geq 10$ regardless of the ODI.

The second night of HSAT monitoring serves only as a backup in case the first night is inadequate to determine the AHI and ODI. Variables derived from HSAT include AHI, ODI, $\mathrm{SaO}_{2}$ nadir and time with $\mathrm{SaO}_{2}<90 \%$. Investigators, treating physicians and participants are blinded to these results until after delivery. The only result that is unblinded prior to delivery is the binary 'OSA positive' versus 'OSA negative' HSAT result. Participants with an HSAT that is positive for OSA will proceed to stage 3. All participants from stage 2 are followed through the time of delivery and assessed for maternal and fetal outcomes after delivery (details below).

\section{Stage 3: randomised trial of PAP therapy}

Participants diagnosed with OSA in stage 2 proceed to stage 3 , which is a randomised controlled trial (RCT) of PAP versus standard care control. The RCT is designed in accordance with the Consolidated Standards of Reporting Trials statement (http://www.consort-statement.org). The primary outcome is unadjusted birth weight.

Participants randomised to PAP are asked to use PAP whenever sleeping, from the time of randomisation until delivery. The PAP device is the ResMed AirSense 10 'AutoSet for Her', set at the widest pressure range of 4-20 $\mathrm{cmH}_{2} \mathrm{O}$. The low end of the range is increased if the participant indicates discomfort from a sensation of insufficient air pressure. During set-up, participants select from full face mask, nasal mask or nasal pillow interfaces, depending on comfort and fit. Initial set-up is performed by a team member with clinical experience with PAP treatment. Participants can change their mask interfaces ad libitum. Adherence to PAP is continuously assessed remotely using a cloud-based monitoring system, 
Airview (ResMed). In case of malfunction of this system, adherence is also recorded by a memory card in each PAP device. Adherence is monitored twice in the first week, then weekly until the end of the study. We have defined acceptable adherence to PAP as $\geq 4$ hours of treatment on $\geq 70 \%$ of nights from the first night after PAP set-up to the night prior to delivery. This definition is based on the Centers for Medicare and Medicaid Services guideline. ${ }^{43}$ If a participant does not meet this benchmark based on the nights studied, she receives a call from a team member and is offered a troubleshooting visit to reassess mask fit, machine settings and other changes in equipment in order to try to improve adherence.

\section{Recruitment, randomisation and blinding}

Participant recruitment occurs simultaneously at all sites (Barnes-Jewish Hospital, Washington University in St Louis; Strong Memorial Hospital, University of Rochester; and Hadassah Hebrew University Medical Center, Jerusalem, Israel). Randomised allocation in stage 3 occurs only after participant enrolment to avoid selection bias. Randomised allocation is performed centrally by random number generator using block randomisation with 1:1 allocation to PAP versus non-PAP control, using randomised blocks of either two or four participants. Randomisation is stratified by study site; within each site, blocks are trichotomised by AHI into low (AHI 5-15), medium (AHI 16-30) or high (AHI >30) as defined above. Only the coordinating-centre study coordinator is unblinded to absolute AHI or AHI category. All sites produce a weekly report on the number of eligible participants, the number of participants approached and the number recruited. To aid data homogeneity, a central data entry system is used (Research Electronic Data Capture; REDCap). At each centre, the investigators have a secure password allowing access to the protected database. After randomised allocation, the participant is notified of her allocation by the site investigator; subjects in the PAP group are then instructed on PAP use and a mask fitting session is scheduled. Participants are instructed not to reveal their group allocation to the technicians performing follow-up ultrasounds or to the obstetricians making clinical management decisions.

\section{Outcome measures and analysis}

\section{Primary outcome}

The goal for stage 1 is to identify OSA risk as defined above for the purpose of recruiting participants into stage 2. The goal for stage 2 is to identify OSA based on HSAT criteria as defined above for the purpose of recruiting participants into stage 3 . The goal for stage 3 , and the primary outcome of the SAFER study, is the impact of the randomised treatment intervention (PAP vs standard care control) on unadjusted birth weight. Birth weight is an endpoint driven by multiple factors including intrauterine fetal growth velocity and gestational age at delivery. These factors may not be independent of each other, as frequently women with severe FGR have interventional delivery performed remote from term, either for fetal indications or for maternal indications (typically when FGR is accompanied by pre-eclampsia). The primary endpoint, unadjusted birth weight, will be corrected for gestational age and by appropriate racial and local nomograms in multivariate analysis.

\section{Secondary outcomes}

The secondary outcome of stage 1 is the percentage of patients with FGR who screen positive for OSA. The secondary outcome for stage 2 is the percentage of patients who test positive for OSA in HSAT. These outcomes will help determine the clinical feasibility and cost-effectiveness of this strategy in the general population. An additional secondary outcome of stage 2 is to assess the predictive value of each of the OSA screening tools for identifying OSA in pregnancy. Secondary outcomes of stage 3 are as follows: (1) obstetric outcomes: ultrasound fetal growth velocity, ${ }^{44}$ enrolment-to-delivery interval, gestational age at delivery, birth weight (as a Z-score) corrected for gestational age ${ }^{45}$ using an algorithm to standardise gestational age, ${ }^{46}$ stillbirth; (2) neonatal outcomes: Apgar score, rate of admission to higher levels of care (NICU or special care nursery), length of neonatal stay.

Exploratory outcomes of stage 3 are as follows: (1) obstetric outcomes: mode of delivery, umbilical cord gases, umbilical artery blood flows; (2) maternal outcomes: postpartum depression Edinburgh Postnatal Depression Scale (EPDS) score, rate of hypertensive disorders of pregnancy, rate of gestational diabetes mellitus; (3) neonatal outcomes: postnatal hypoglycaemia $(<40 \mathrm{mg}$ / $\mathrm{dL}$ at any time $)^{47}$ and/or requirement for intravenous glucose treatment based on American Academy of Pediatrics guidelines, ${ }^{48}$ rates of hypoxic ischaemic neonatal encephalopathy, neonatal death in first year of life.

Primary and secondary outcomes are compared between the two study groups in stage 3 (OSA-positive patients randomised to standard care with no PAP vs those randomised to PAP therapy). We use intention-totreat analysis.

\section{Planned subgroup analyses}

We plan the following subgroup analyses:

\section{PAP use}

The effect of PAP on primary and secondary outcomes based not on intention to treat, but rather on PAP adherence (as described above). The clinical justification of this subgroup allocation is to assess whether a negative or a borderline primary outcome of SAFER may mask a true clinical effect of PAP when used with good adherence. This subgroup analysis will also accommodate the unlikely crossover of a patient allocated to the no-PAP control in stage 3, but who is subsequently referred by their primary physician or obstetrician for OSA work-up (not current standard of care) and who goes on to receive PAP. 


\section{OSA severity}

The effect of PAP on primary and secondary outcomes with stratification by OSA severity (mild, AHI 5-15; moderate, AHI 15-30; severe $>30$ ). We hypothesise that maternal and fetal outcomes will be most improved following PAP therapy in patients with the most severe cases of OSA. The clinical justification of this subgroup allocation is to assess whether a negative or borderline primary outcome of SAFER may mask a true clinical effect of PAP when used in patients with more severe OSA; this may also aid in the determination of an optimal target population based on OSA severity for clinical use of PAP in FGR.

\section{Planned exploratory subgroup analyses PAP use}

The effect of PAP usage as a continuous variable (mean hours of PAP use per night) on primary and secondary outcomes; the purpose of this exploratory subgroup analysis is to explore the possible dose-response effect of PAP.

\section{OSA severity}

The effect of PAP on primary and secondary outcomes with stratification by secondary measures from the HSAT: ODI, $\mathrm{SpO}_{2}$ nadir and time with $\mathrm{SpO}_{2}<90 \%$; where the stratification into mild, moderate and severe will be determined after examining the distribution of data; the purpose of this exploratory subgroup analysis is to explore whether other measures of OSA severity, particularly oxygen desaturation, are useful to direct which patients may benefit from PAP therapy.

\section{Effect of untreated OSA}

Comparison between OSA-negative patients from stage 2 versus untreated OSA-positive patients (non-PAP control) from stage 3 (we anticipate this will be approximately a $3: 1$ ratio); the purpose of this exploratory subgroup analysis is to assess the deleterious effects of OSA on maternal and fetal outcomes in these patients.

\section{Statistical analysis}

Data are assessed for normality by visual inspection of the frequency plot (histogram) and Q-Q plot of each outcome variable and by the Kolmogorov-Smirnov test. Normally distributed data are presented as mean (SD); non-normally distributed data are presented as median (IQR). Our primary outcome (birth weight) (derived from stage 3 ) is assessed by two-tailed parametric t-test (normal distribution) or non-parametric Wilcoxon rank-sum test (non-normal distribution) as appropriate, using intention to treat as the grouping variable. The same tests are used for other continuous secondary outcome variables (gestational age at delivery; enrolment-to-delivery interval; birth weight corrected for gestational age; Apgar and umbilical cord gas). For categorical outcomes (higher level nursery admission; stillbirth; compliance with PAP), the $\chi^{2}$ test or Fisher's exact test (if expected cells are small) is used as appropriate. Additionally, we use a mixed effects regression model to assess the effect of PAP on the longitudinal association between estimated fetal weight and Doppler ultrasound umbilical artery flow, while controlling for maternal, obstetric and fetal variables that may affect the primary outcome. Only the intercept will be specified as the random component to account for any omitted variable. By standard convention, statistical significance is based on a two-sided $p$ value $<0.05$ to determine the significance of association. Statistical testing is currently planned to use SAS V.9.4 (SAS Institute).

\section{Sample size calculation}

The baseline birth weight of infants with FGR in the population from our primary study centre (Barnes-Jewish Hospital, St Louis, Missouri) is $2535 \pm 234 \mathrm{~g}$ (unpublished data). Based on these data, we calculated that 104 evaluable participants with OSA will need to be randomised to either PAP or control (stage 3) in order to have $90 \%$ power to detect a $150 \mathrm{~g}$ difference in birth weight in the PAP group compared with control. This is based on an alpha of 0.05 , anticipated $5 \%$ loss to follow-up and a twotailed t-test.

The risk of OSA in high-risk obstetric populations (such as FGR) is as high as $35 \% .{ }^{49}$ As we are screening participants for OSA risk by a brief telephone or in-person questionnaire (stage 1) prior to HSAT testing (stage 2), we estimate that there will be an OSA-positive HSAT in $30 \%-50 \%$ of these screened participants. Consequently, we estimate that we will need to assess HSAT in 200-350 participants in stage 2 in order to achieve our sample size of 104 evaluable participants for stage 3 . We estimate needing to screen some 500-1000 participants in stage 1 in order to identify these 200-350 participants for stage 2. Based on an estimated combined total of 20000 births annually with an estimated incidence of $15 \%$ of FGR at the above centres, we estimate 3000 pregnancies complicated by FGR each year. Accounting for participants who will not meet study inclusion criteria, or will decline to participate, the recruitment process is expected to last approximately 2 years.

\section{Analysis of pragmatic elements of the SAFER study}

The SAFER study is a pragmatic trial. The pragmatic elements of the study were quantified using the Pragmatic Explanatory Continuum Indicator Summary-2 tool (https://www.precis-2.org). Data were obtained from the principal investigators in all study centres. According to six of the nine criteria, the SAFER study is largely a pragmatic study (figure 2 ). The study is particularly pragmatic for experimental intervention (a standard commercially available product), follow-up, relevance of clinical outcomes and analysis. It is more explanatory for patient selection/recruitment and organisational intervention. This is because OSA work-up is not currently a standard of care for FGR and patients only reach stage 3 if they have FGR with HSAT-verified OSA. In this way, the study targets patients most likely to experience benefits in fetal 


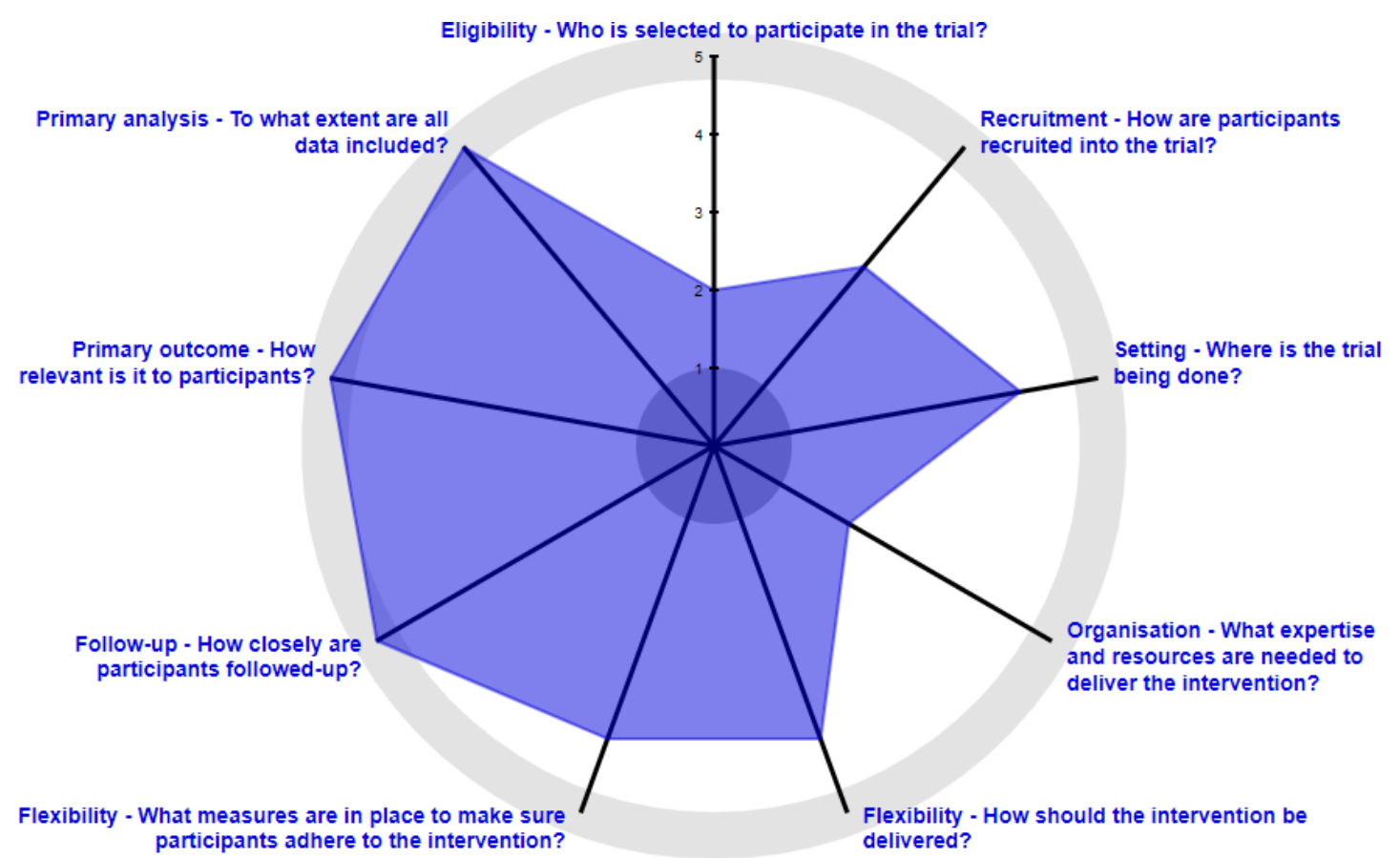

Figure 2 Pragmatic Explanatory Continuum Indicator Summary-2 (PRECIS-2) wheel for characterising the degree to which elements of a study are more explanatory (toward the centre of the wheel) or pragmatic (toward the periphery).

growth from PAP, but the generalisability to other populations may be more limited.

\section{Strengths and limitations}

The SAFER study has important strengths. It is a multicentre study of a safe, routinely available intervention that examines an easily measured outcome with important short-term and long-term ramifications for the well-being of neonates and possibly for later adult life. SAFER is a pragmatic study and the intervention does not preclude any existing approaches for the management of these high-risk pregnancies. As PAP is worn only when sleeping, typically at home, it is relatively easy to keep the research team and clinical providers blinded to group allocation. As the PAP device used in this study records PAP compliance, we do not have to rely on compliance self-reporting which may overestimate nightly device use.

The SAFER study has several limitations. There is currently no definitive estimate for the impact of PAP on birth weight; hence, the effect measure used in the sample size estimation may be inaccurate, which may affect the study's power in regard to our primary and secondary endpoints. It is likely that follow-up studies will be warranted, especially regarding whether or not the results from this specific target population can be generalised to other pregnant women with FGR, or pregnant women with OSA who may have pregnancy complications without FGR, for example, pre-eclampsia. It is the intention of the investigators to follow-up a positive result (that PAP increases intrauterine fetal growth) with a larger study to assess long-term neonatal neurological outcome.

Estimates of fetal weight were all based on the Hadlock formula using the standard biometric parameters (biparietal diameter, head circumference, abdominal circumference, femur length) $[52,53] .{ }^{50} 51$ However, when converting fetal weight to a calculated percentile, this must reference a 'population or customized standard' ${ }^{52}$ As this is a pragmatic multicentre study, each study centre defines FGR and SGA by the population or customised standard used in their routine care (see table 1). This will lead to a slight difference between centres in FGR and SGA definition. However, fetal growth varies between different ethnic and geographical populations, so a single absolute imposed standard is of limited relevance clinically. The impact of this factor in stage 3 is reduced by stratification of randomised allocation by study centre.

Although investigators, treating physicians and ultrasound technicians are blinded to participant group allocation in this study, we do not blind participants. We are confident that our primary outcome and all of the secondary outcome measures (with the exception of the EPDS) can be assessed blindly, which minimises the risk of bias. We considered using sham PAP as a placebo control. While this could minimise bias in evaluating the effect of PAP, wearing an ineffective mask does increase the risk of discomfort and sleep disturbances for the control group, which potentially biases the results toward the active group. A recent study ${ }^{53}$ demonstrated that there was no difference in outcome between sham PAP and a device-free control; however, most subjects in the sham PAP group guessed correctly that they were receiving the placebo intervention.

Home sleep testing may underestimate the true presence of SDB for several reasons, most notably because it 
uses the study duration as the denominator to calculate AHI. In addition, in this study, our protocol may be overreliant on oxygen desaturation, as that feature may not be as prominent in a young female pregnant population. ${ }^{50}$ Hence, our methods may underestimate the true prevalence and severity of SDB, and preferentially select the study population with more severe SDB. This selected population with more severe SDB may have a greater likelihood of exhibiting a clinical effect with PAP treatment. However, in this pragmatic study, we need to use HSAT so that the intervention can be started within as narrow a time frame as possible.

This study uses intention-to-treat analysis to assess the efficacy of the intervention. The adherence to PAP is relatively low across all populations. ${ }^{51}{ }^{54}$ Adherence is generally higher for aPAP devices, as used in this study, when compared with CPAP devices. ${ }^{51}$ Two recent large national database studies reported conflicting results regarding gender effects on PAP adherence-either lower in men ${ }^{51}$ or lower in women, ${ }^{54}$ although it is not known whether any of these women were pregnant in those studies. It is possible that pregnant women concerned about potentially optimising fetal and maternal outcomes would be more adherent to PAP. We performed a pilot study in preparation for this protocol (see the Patient and public involvement section) in which we observed PAP adherence in seven out of nine subjects tested. Our calculated power does not specifically accommodate non-adherence. Accordingly, we also describe a planned subgroup analysis in which PAP adherence is used rather than intention to treat. Ultimately, this pragmatic study can only assess whether administering a PAP device will improve outcomes in pregnancy. If PAP is not effective during pregnancy because women do not adhere to it, that raises a separate question that will require addressing separately.

\section{Potential benefits, risks and alternatives \\ Benefits}

All participants reaching stage 2 of the study will potentially benefit from being identified as at risk for OSA and being referred to their primary care physician for evaluation by a sleep physician after delivery.

\section{Risks}

The likelihood of adverse events in this study is low as the devices that will be used in the study (HSAT and aPAP) are ones that have been used in the general and obstetric population for years. In the unlikely event that serious side effects occur, these will be documented and reported to the human research protection office and to the study's Data Safety Monitor.

\section{Minimisation of risks to confidentiality}

All participants are assigned a unique study ID number. The link to identifiers will be destroyed at the end of the study. Data will be stored under lock and key (office, file cabinet) and only the investigators and research team will have access. If data are published, there will be no link to identifiers. Study data are not entered into participants' medical records. Data regarding PAP compliance are downloaded from ResMed's secure, cloud-based patient management system to a secure server at the primary site (Washington University), where they are stored for the duration of the study.

Data from this study will be recorded using REDCap, a web-based, Health Insurance Portability and Accountability Act-compliant application. Access to the data is password protected. REDCap servers are housed in a secure data centre and information transmission is encrypted.

\section{Adverse event reporting and safety monitoring}

The research team continuously monitors the study for adverse events. All serious adverse events (SAEs) are reported to the Institutional Review Board (IRB) according to IRB stipulations. Additionally, an attending anaesthesiologist at Washington University who is not involved in the study serves as the Data Safety Monitor; given the small size and relatively low-risk nature of the protocol, an individual physician rather than a full Data Safety Monitoring Board is used. This monitor reviews all adverse events annually and reviews SAEs or unexpected adverse events as they occur.

\section{Premature study termination}

The only interim analysis to be performed in this study will be a single, blinded analysis performed by the Data Safety Monitor at the mid-way point, after 52 patients have completed stage 3. This interim analysis will assess differences between groups in birth weight, gestational age at delivery, intrauterine fetal death or reported major adverse events. The study code will not be broken, and other secondary endpoints will not be assessed. If there is a statistically significant difference between groups in one of these selected outcomes, then the Data Safety Monitor will break the code. If the intervention is associated with smaller birth weight, lower gestational age at delivery, more intrauterine fetal death or more reported major adverse events, then this will be brought to the attention of the investigators and the primary study site IRB, for consideration of possible premature termination of the study on the grounds of increased harm due to the intervention. There will be no premature termination of the study due to increased benefit of the intervention. Unless there is an increased harm of the intervention, the Data Safety Monitor will not give any information to the investigators regarding the interim analysis.

\section{Ethics and dissemination}

Informed consent for stage 1 may be by telephone, electronic or written consent. Informed consent for stages 2 and 3 is obtained by a study investigator using electronic or written consent (see online supplemental file 1). There are no additional risks associated with the screening study (stage 1) or the observational HSAT study (stage 2). There 
is a potential ethical concern regarding randomisation of HSAT-positive participants (identified from stage 2 as having OSA) to either a PAP (intervention) or a no-PAP (control) group. However, this concern is minimised by the following arguments: (A) HSAT, or a formal sleep study, is not a standard care for investigating FGR, and the diagnosis of OSA is rarely made in pregnancy; hence, the diagnosis is only made as a result of this study ${ }^{2324}$; (B) PAP is not a standard care for sleep disturbance in pregnancy as many practitioners and participants rightly or wrongly assume that this will improve after delivery, ${ }^{24}$ while PAP is seen as a long-term therapeutic intervention. After delivery, participants with AHI $\geq 5$ will be given a standard IRB-approved letter along with their HSAT results. This information can be presented to their primary care physician for possible referral to a sleep specialist.

The trial steering committee is responsible for all major decisions regarding changes to the protocol. The committee communicates these changes to the IRB, the trial registry and appropriate parties. Data will be shared in keeping with the data sharing statement below. Dissemination plans include presentations at scientific conferences. The results of the SAFER trial will be published in a peer-reviewed journal. Dissemination of results to study participants and their family members will be available on request. Updates and results of the study will be available to the public at ClinicalTrials.gov.

\section{Author affiliations}

${ }^{1}$ Anesthesiology, Washington University in St Louis School of Medicine, St Louis,

Missouri, USA

${ }^{2}$ Anesthesiology, University of Rochester Medical Center, Rochester, New York, USA ${ }^{3}$ Obstetrics and Gynecology, Washington University in St Louis School of Medicine, St Louis, Missouri, USA

${ }^{4}$ Obstetrics and Gynecology, Hadassah University Hospital, and the Faculty of Medicine, Hebrew University of Jerusalem, Jerusalem, Israel

${ }^{5}$ Neonatology, Washington University in St Louis School of Medicine, St Louis, Missouri, USA

${ }^{6}$ Neurology, Washington University in St Louis School of Medicine, St Louis, Missouri, USA

${ }^{7}$ Anesthesiology, Critical Care and Pain Medicine, and the Wohl Institute of Translational Medicine, Hadassah University Hospital and the Faculty of Medicine, Hebrew University of Jerusalem, Jerusalem, Israel

Correction notice This article has been corrected since it was first published. The study group name in the author list 'the SAFER study group' has been added.

Acknowledgements We acknowledge our multidisciplinary study collaborators in the SAFER study group. Obstetrics: Washington University in St Louis, Missouri: EC. University of Rochester, New York: Loralei Thornburg. Hadassah Hebrew University, Jerusalem, Israel: Simcha Yagel, SP, Joshua Rosenbloom, Doron Cabiri, Avraham Nahum, Shlomo Yahalomy, David Shviki. Sleep Medicine: Washington University in St Louis, Missouri: YESJ. University of Rochester, New York: Lynn Liu. Hadassah Hebrew University, Jerusalem, Israel: Neville Birkman, Yoel Reiter. Neonatology: Washington University in St Louis, Missouri: BW. Hadassah Hebrew University, Jerusalem, Israel: Smadar Even-Tov Friedman, Noa Ofek. Study coordinators: Washington University in St Louis, Missouri: EW, Catherine Dalton. University of Rochester, New York: Tammy Ortiz, Nancy Robertson, Janet Vaughan. Hadassah Hebrew University, Jerusalem, Israel: Batya Nachmani, Esty Gold.

Contributors Authorship for this study is given to key personnel involved in study design, recruitment, data collection and data analysis. There are no publication restrictions and no professional writers involved in the generation of the manuscript. AH is the site principle investigator for Washington University. $\mathrm{JN}$ is the site principle investigator for the University of Rochester. SK jointly conceived the study together with YG and EML. EC and SP are the overall advisers for maternalfetal medicine. BW is the overall adviser for neonatal medicine. YESJ is the overall adviser for sleep medicine. ABA is the statistical consultant. EW is the overall study coordinator. EML is the overall study principle investigator, and jointly conceived the study together with YG and SK. YG is the site principle investigator for Hadassah Hebrew University, and jointly conceived the study together with SK and EML. YG, SK and EML wrote the grant that led to this study, and all authors jointly wrote this manuscript. All authors agree to be accountable for the accuracy and integrity of all aspects of the SAFER trial.

Funding The investigators YG, EML and SK were the initiators and sponsors of this study. The contact sponsor is YG (see contact details under corresponding author details). This study was supported by a competitive research grant from the ResMed Research Foundation (Clinical Research Grant); EML as principle investigator, YG and SK as coinvestigators. The Washington University School of Medicine (WUSM) also sponsored part of the pilot and run-in costs (YG, principle investigator). Home sleep apnoea testing devices and autoregulated positive airway pressure devices were provided gratis by the ResMed.

Disclaimer The initiation, design, management, analysis and reporting of the study are entirely independent of both ResMed Research Foundation and ResMed Corporation, and the sole responsibility of the investigators.

Competing interests None declared.

Patient consent for publication Not required.

Provenance and peer review Not commissioned; externally peer reviewed.

Supplemental material This content has been supplied by the author(s). It has not been vetted by BMJ Publishing Group Limited (BMJ) and may not have been peer-reviewed. Any opinions or recommendations discussed are solely those of the author(s) and are not endorsed by BMJ. BMJ disclaims all liability and responsibility arising from any reliance placed on the content. Where the content includes any translated material, BMJ does not warrant the accuracy and reliability of the translations (including but not limited to local regulations, clinical guidelines, terminology, drug names and drug dosages), and is not responsible for any error and/or omissions arising from translation and adaptation or otherwise.

Open access This is an open access article distributed in accordance with the Creative Commons Attribution Non Commercial (CC BY-NC 4.0) license, which permits others to distribute, remix, adapt, build upon this work non-commercially, and license their derivative works on different terms, provided the original work is properly cited, appropriate credit is given, any changes made indicated, and the use is non-commercial. See: http://creativecommons.org/licenses/by-nc/4.0/.

ORCID iD

Yehuda Ginosar http://orcid.org/0000-0003-2258-4935

\section{REFERENCES}

1 Sharma D, Shastri S, Sharma P. Intrauterine growth restriction: antenatal and postnatal aspects. Clin Med Insights Pediatr 2016;10:CMPed.S40070.

2 American College of Obstetricians and Gynecologists Committee on Practice Bulletins-Obstetrics and the Society forMaternalFetalMedicin. ACOG practice Bulletin No. 204: fetal growth restriction. Obstet Gynecol 2019:133-109.

3 Barker DJP. Adult consequences of fetal growth restriction. Clin Obstet Gynecol 2006;49:270-83.

4 Pallotto EK, Kilbride HW. Perinatal outcome and later implications of intrauterine growth restriction. Clin Obstet Gynecol 2006;49:257-69.

5 Fetal growth restriction: ACOG practice Bulletin, number 227. Obstet Gynecol 2021:137:e16-28.

6 Mari G, Hanif F. Intrauterine growth restriction: how to manage and when to deliver. Clin Obstet Gynecol 2007;50:497-509.

7 Hankins GDV, Speer M. Defining the pathogenesis and pathophysiology of neonatal encephalopathy and cerebral palsy. Obstet Gynecol 2003:102:628-36.

8 Fang S. Management of preterm infants with intrauterine growth restriction. Early Hum Dev 2005;81:889-900.

9 Pien GW, Schwab RJ. Sleep disorders during pregnancy. Sleep 2004;27:1405-17.

10 Peppard PE, Young T, Barnet JH, et al. Increased prevalence of sleep-disordered breathing in adults. Am J Epidemiol 2013;177:1006-14.

11 MedicineA.A.o.SDarien IL. International Classification of Sleep Disorders, 2014. 
12 Iber C, Westchester IL. The American Academy of Sleep Medicine Manual for the scoring of sleep and associated events:rulesterminology and technical specifications, 2007

13 Louis J, Auckley D, Miladinovic B, et al. Perinatal outcomes associated with obstructive sleep apnea in obese pregnant women. Obstet Gynecol 2012;120:1085-92.

14 Chen $\mathrm{Y}-\mathrm{H}$, Kang J-H, Lin C-C, et al. Obstructive sleep apnea and the risk of adverse pregnancy outcomes. Am J Obstet Gynecol 2012;206:136.e1-136.e5.

15 Pamidi S, Pinto LM, Marc I, et al. Maternal sleep-disordered breathing and adverse pregnancy outcomes: a systematic review and metaanalysis. Am J Obstet Gynecol 2014;210:p. 52 e1-52.

16 Izci-Balserak B, Pien GW. Sleep-Disordered breathing and pregnancy: potential mechanisms and evidence for maternal and fetal morbidity. Curr Opin Pulm Med 2010;16:574-82.

17 Louis JM, Mogos MF, Salemi JL, et al. Obstructive sleep apnea and severe maternal-infant morbidity/mortality in the United States, 19982009. Sleep 2014;37:843-9.

18 Izci Balserak B, Jackson N, Ratcliffe SA, et al. Sleep-Disordered breathing and daytime napping are associated with maternal hyperglycemia. Sleep Breath 2013;17:1093-102.

19 Facco FL, Ouyang DW, Zee PC, et al. Sleep disordered breathing in a high-risk cohort prevalence and severity across pregnancy. $A m \mathrm{~J}$ Perinatol 2014;31:899-904.

20 Facco FL, Ouyang DW, Zee PC, et al. Development of a pregnancyspecific screening tool for sleep apnea. J Clin Sleep Med 2012;8:389-94.

21 Olivarez SA, Ferres M, Antony K, et al. Obstructive sleep apnea screening in pregnancy, perinatal outcomes, and impact of maternal obesity. Am J Perinatol 2011;28:651-8.

22 Facco FL. Sleep-Disordered breathing and pregnancy. Semin Perinatol 2011;35:335-9.

23 Dominguez JE, Lockhart EM, Miskovic A, et al. Recognition of obstructive sleep apnea in pregnancy survey. Int J Obstet Anesth 2016;26:85-7.

24 Karan S, Ginosar Y. Gestational sleep apnea: have we been caught napping? Int J Obstet Anesth 2016;26:1-3.

25 Lavie L. Oxidative stress in obstructive sleep apnea and intermittent hypoxia--revisited--the bad ugly and good: implications to the heart and brain. Sleep Med Rev 2015;20:27-45.

26 Ginosar Y, Gielchinsky Y, Nachmansson N, et al. BOLD-MRI demonstrates acute placental and fetal organ hypoperfusion with fetal brain sparing during hypercapnia. Placenta 2018;63:53-60.

27 Sahin FK, Koken G, Cosar E, et al. Obstructive sleep apnea in pregnancy and fetal outcome. Int J Gynaecol Obstet 2008;100:141-6.

28 Olivarez SA, Maheshwari B, McCarthy M, et al. Prospective trial on obstructive sleep apnea in pregnancy and fetal heart rate monitoring. Am J Obstet Gynecol 2010;202:552.e1-552.e7.

$29 \mathrm{Xu} \mathrm{T}$, Li T, Wei D, et al. Effect of automatic versus fixed continuous positive airway pressure for the treatment of obstructive sleep apnea: an up-to-date meta-analysis. Sleep Breath 2012;16:1017-26.

30 Guo J, Sun Y, Xue L-J, et al. Effect of CPAP therapy on cardiovascular events and mortality in patients with obstructive sleep apnea: a meta-analysis. Sleep Breath 2016;20:965-74.

31 Qureshi WT, Nasir UB, Alqalyoobi S, et al. Meta-Analysis of continuous positive airway pressure as a therapy of atrial fibrillation in obstructive sleep apnea. Am J Cardiol 2015;116:1767-73.

32 Donovan LM, Boeder S, Malhotra A, et al. New developments in the use of positive airway pressure for obstructive sleep apnea. $J$ Thorac Dis 2015;7:1323-42.

33 Feng Y, Zhang Z, Dong Z-zhou. Effects of continuous positive airway pressure therapy on glycaemic control, insulin sensitivity and body mass index in patients with obstructive sleep apnoea and type 2 diabetes: a systematic review and meta-analysis. NPJ Prim Care Respir Med 2015;25:15005.

34 Sassani A, Findley LJ, Kryger M, et al. Reducing motor-vehicle collisions, costs, and fatalities by treating obstructive sleep apnea syndrome. Sleep 2004;27:453-8.

35 Blyton DM, Sullivan CE, Edwards N. Reduced nocturnal cardiac output associated with preeclampsia is minimized with the use of nocturnal nasal CPAP. Sleep 2004;27:79-84.

36 Edwards N, Blyton DM, Kirjavainen T, et al. Nasal continuous positive airway pressure reduces sleep-induced blood pressure increments in preeclampsia. Am J Respir Crit Care Med 2000;162:252-7.

37 Chan A-W, Tetzlaff JM, Altman DG, et al. Spirit 2013 statement: defining standard protocol items for clinical trials. Ann Intern Med 2013;158:200-7.
38 Lockhart EM, Ben Abdallah A, Tuuli MG, et al. Obstructive sleep apnea in pregnancy: assessment of current screening tools. Obstet Gynecol 2015;126:93-102.

39 Izci-Balserak B, Zhu B, Gurubhagavatula I, et al. A screening algorithm for obstructive sleep apnea in pregnancy. Ann Am Thorac Soc 2019;16:1286-94.

40 Phurrough S JL, Stiller J, Brechner R. Decision Memo for SLEEP TESTING for Obstructive SLEEP Apnea (OSA) (CAG-00405N), 2009. C.f.M.a.M.Government services us

41 Berry RB, Budhiraja R, Gottlieb DJ, et al. Rules for scoring respiratory events in sleep: update of the 2007 AASM manual for the scoring of sleep and associated events. deliberations of the sleep apnea definitions Task force of the American Academy of sleep medicine. $J$ Clin Sleep Med 2012;8:597-619.

42 Kapur VK, Auckley DH, Chowdhuri S, et al. Clinical practice guideline for diagnostic testing for adult obstructive sleep apnea: an American Academy of sleep medicine clinical practice guideline. J Clin Sleep Med 2017:13:479-504.

43 American Hospital Association. Positive Airway Pressure (PAP) Devices for the Treatment of Obstructive Sleep Apnea 2020.

44 Grantz KL, Kim S, Grobman WA, et al. Fetal growth velocity: the NICHD fetal growth studies. Am J Obstet Gynecol 2018;219:p. 285 e1-285.

45 Oken $\mathrm{E}$, Kleinman KP Rich-Edwards J, et al. A nearly continuous measure of birth weight for gestational age using a United States national reference. BMC Pediatr 2003;3:6.

46 Novak GP, Kurtzberg D, Kreuzer JA, et al. Cortical responses to speech sounds and their formants in normal infants: maturational sequence and spatiotemporal analysis. Electroencephalogr Clin Neurophysiol 1989;73:295-305.

47 Smith GCS. Antenatal betamethasone for women at risk for late preterm delivery. N Engl J Med 2016;375:486.

48 Adamkin DH, Committee on Fetus and Newborn. Postnatal glucose homeostasis in late-preterm and term infants. Pediatrics 2011:127:575-9.

49 Facco FL, Ouyang DW, Zee PC, et al. Sleep disordered breathing in a high-risk cohort prevalence and severity across pregnancy. Am J Perinatol 2014;31:899-904.

50 Bourjeily G, Fung JY, Sharkey KM, et al. Airflow limitations in pregnant women suspected of sleep-disordered breathing. Sleep Med 2014:15:550-5.

51 Pandey A, Mereddy S, Combs D, et al. Socioeconomic inequities in adherence to positive airway pressure therapy in population-level analysis. J Clin Med 2020;9. doi:10.3390/jcm9020442. [Epub ahead of print: 0602 2020].

52 McCowan LM, Figueras F, Anderson NH. Evidence-Based national guidelines for the management of suspected fetal growth restriction: comparison, consensus, and controversy. Am J Obstet Gynecol 2018;218:S855-68.

53 Reid ML, Gleason KJ, Bakker JP, et al. The role of sham continuous positive airway pressure as a placebo in controlled trials: best apnea interventions for research trial. Sleep 2019;42. doi:10.1093/sleep/ zsz099. [Epub ahead of print: 0108 2019].

54 Patel SR, Bakker JP, Stitt CJ, et al. Age and sex disparities in adherence to CPAP. Chest 2021;159:382-9.

55 Hadlock FP, Deter RL, Harrist RB, et al. Estimating fetal age: computer-assisted analysis of multiple fetal growth parameters. Radiology 1984;152:497-501.

56 Alexander GR, Himes JH, Kaufman RB, et al. A United States national reference for fetal growth. Obstet Gynecol 1996;87:163-8.

57 Fenton TR, Kim JH. A systematic review and meta-analysis to revise the Fenton growth chart for preterm infants. BMC Pediatr 2013;13:59.

58 Hadlock FP, Harrist RB, Sharman RS, et al. Estimation of fetal weight with the use of head, body, and femur measurements--a prospective study. Am J Obstet Gynecol 1985;151:333-7.

59 Sheth T, Glantz JC. Third-Trimester fetal biometry and neonatal outcomes in term and preterm deliveries. J Ultrasound Med 2016;35:103-10.

60 Durie DE, Thornburg LL, Glantz JC. Effect of second-trimester and third-trimester rate of gestational weight gain on maternal and neonatal outcomes. Obstet Gynecol 2011;118:569-75.

61 Stirnemann J, Villar J, Salomon LJ, et al. International estimated feta weight standards of the INTERGROWTH-21st Project. Ultrasound Obstet Gynecol 2017;49:478-86.

62 Dollberg S, Haklai Z, Mimouni FB. Birth weight standards in the liveborn population in Israel. Isr Med Assoc J 2005;7:311-4. 